\title{
Thermodynamic Performance and Optimal Analysis of A Multi-Level Quantum Dot Thermal Amplifier
}

Wei Li, Yunyun Yang, Jing Fu, Zebin Lin and Jizhou He*

A multi-level quantum dot thermal amplifier with heat leakage is proposed. Based on the theory of the Landauer formula, the electron flux, the heat flux, the heat-pumping rate and the coefficient of performance are derived. The three-dimensional projection graphs of the heat-pumping rate and the coefficient of performance versus the relative central energy levels are plotted. Moreover, the influence of the energy-level spacing, the number of energy levels, the total coupling strength, the asymmetry factor and the heat leakage coefficient on the optimal performance parameters is analyzed in detail. By choosing appropriate values of the system parameters, one can enhance the maximum heat-pumping rate and coefficient of performance.

Keywords: Thermal amplifier; Multi-level quantum dot; Irreversible thermodynamics; Performance optimization

Received 8 October 2019, Accepted 9 December 2019

DOI: $10.30919 /$ esee 8 c362

\section{Introduction}

The thermoelectric devices can be functioned as heat engines, refrigerators, heat pumps, and thermal amplifiers by the transport of electrons. Recently, the studies of three-terminal thermoelectric devices with practical nanostructures such as quantum-dot, nanowire, quantum-well and superlattice, have attracted great interest. And they provide solutions to the problem of low efficiency of conventional thermoelectric devices.

For example, Edwards et al investigated theoretically the quantum-dot refrigerator (QDR) which utilizes the discrete energy levels of quantum dots to customize the electronic Fermi-Dirac distribution, cooling a small reservoir to far below the ambient temperature. ${ }^{1,2}$ Prance et al presented experimentally measurements of a QDR designed to cool a $6 \mu \mathrm{m}^{2}$ electron gas, and significant electro-static interactions were observed in this device. ${ }^{3}$ Jordan et al. proposed a threeterminal heat engine with resonant-tunneling dots and obtained the maximum power and the corresponding efficiency. ${ }^{4}$ Sothmann et al. proposed a three-terminal heat engine based on resonant-tunneling wells and obtained the maximum power and the corresponding efficiency. ${ }^{5}$ Choi et al. analyzed the performance of a three-terminal heat engine for energy harvesting and a refrigerator for cooling purpose based on semiconductor superlattices in which the periodicity of the superlattice structure creates an energy miniband, and discussed phonon heat current through the system. ${ }^{6}$ Jiang et al. studied thermoelectric three-terminal hopping transport through one-dimensional nano-systems and the near-field inelastic heat engine in the linear-response regime. ${ }^{7,8}$ And Chen et al. analyzed the thermodynamic performance of an irreversible energy selective electron heat engine with double resonances and discussed the impacts of heat leakage and structure parameters of the electron system on the optimal performance of the heat engine. ${ }^{9}$ They also make the optimal analysis for the performance of an irreversible three-electronreservoir energy selective electron cooling device with heat leakage. ${ }^{10} \mathrm{Su}$ et al. analyzed the performances of a refrigerator driven by hot electrons ${ }^{11}$ and Zhang et al. discussed the thermodynamic characteristics of a model of three-terminal coupled quantum-dot refrigerator. ${ }^{12}$ Otherwise, Wang et al. investigated the nonlinear effect of a three-terminal refrigerator. ${ }^{13}$ In addition, Chen et al. put forward the equivalent combined systems of three-heat-source heat pumps and compared the performance of them with the performance of two-heat-source heat pumps. ${ }^{14}$ The relationship between optimal the coefficient of performance (COP) and heating load of a three-heat-reservoir endoreversible heat pump with non- 
linear heat transfer was derived. ${ }^{15}$ The optimal thermodynamic performance of an irreversible three-heat-source heat pump ${ }^{16}$ and the thermodynamic analysis for an irreversible heat pump working on reversed Brayton cycle ${ }^{17}$ were studied. Su et al. and Peng et al. separately investigated thermodynamic performance of thermal amplifiers based on ideal resonanttunneling filters. ${ }^{18-20}$ Other three-terminal thermoelectric devices which are driven by phonons, magnons, photons, also have been analyzed. ${ }^{21-29}$

On the basis of the previous works, we propose a multi-level quantum dot thermal amplifier. The main focus in this paper is to analyze the thermodynamic performance characteristics and the optimal performance of a multi-level quantum dot thermal amplifier. The influence of the main system parameters, including the energy-level spacing, the number of energy levels, the total coupling strength, the asymmetry factor and the heat leakage coefficient on the performance of the thermal amplifier is discussed in detail.

\section{Model Description}

The thermal amplifier considered here which consists of three electron reservoirs connected via two multi-level quantum dots is illustrated in Fig. 1. Such thermal amplifier can be designed for two connective methods, where one is that electrons flow from the high-temperature reservoir to low-temperature reservoir, as shown in Fig. 1(a), the other is that electrons flow from the low-temperature reservoir to high-temperature reservoir, as shown in Fig. 1(b). The temperatures and the chemical potentials of three reservoirs satisfy the following relations: $T_{H}>T_{M}>T_{C}$ and $\mu_{H}=\mu_{C} \equiv \mu_{0}$, while the chemical potential of the reservoir $M$ is determined by the conservation of the electron flux. $\varepsilon_{\mathrm{i}}(i=H, C)$ represent the central energy level of the quantum dot $Q D_{i}$, respectively. The reservoir $H(C)$ can only exchange electrons with quantum dot $Q D_{H}\left(Q D_{C}\right)$ through the energy level $\varepsilon_{H}\left(\varepsilon_{C}\right)$. The energy level $\varepsilon_{H}$ is near the Fermi level of the reservoir $H$, while the energy level $\varepsilon_{C}$ is near the Fermi level of the reservoir $C . \delta \varepsilon$ is the level spacing between two adjacent energy levels in quantum dots. Here the position of each energy level in quantum dot $Q D_{i}$ is assumed to be

$$
\varepsilon_{i, N}=\varepsilon_{i}+j \delta \varepsilon\left\{\begin{array}{l}
j=\frac{N}{2} \quad N: \text { even } \\
j=-\frac{N-1}{2} \quad N: \text { odd }
\end{array}\right.
$$

(a)

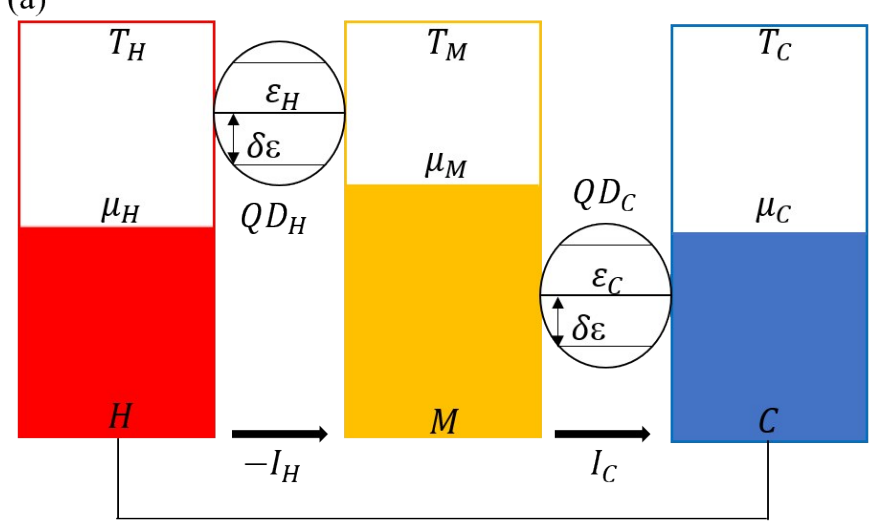

(b)

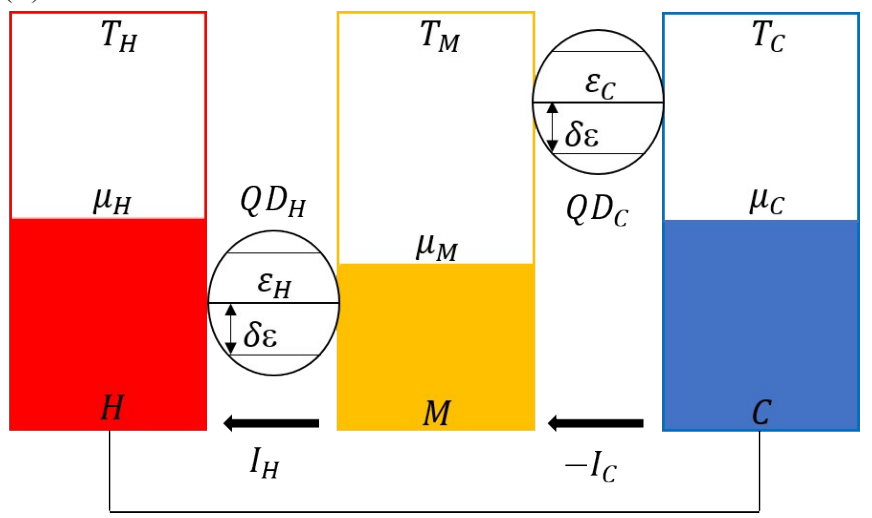

Fig. 1 The schematic diagram of a three-terminal thermal amplifier based on multi-level quantum dots. The $H$ and $C$ electronic terminals are connected by electronic conductors. The positive direction of the electron flux is indicated by the solid black arrow. 
where $N$ is the number of discrete energy levels in the quantum dots. For example, $N=3$, the distribution of the energy levels in the quantum dot is shown in Fig.1.

According to Landauer formula, the electron flux from the reservoir $M$ and the heat flux from the reservoir $i$ are given by

$$
I_{i}=\frac{2}{h} \int_{-\infty}^{+\infty} d \varepsilon T_{e f f i, i}(\varepsilon)\left[f_{M}(\varepsilon)-f_{i}(\varepsilon)\right]
$$

and

$$
\dot{Q}_{i}=\frac{2}{h} \int_{-\infty}^{+\infty} d \varepsilon\left(\varepsilon-\mu_{i}\right) T_{e f f i}(\varepsilon)\left[f_{i}(\varepsilon)-f_{M}(\varepsilon)\right]
$$

where $f_{i}=\left[\exp \left[\left(\varepsilon-\mu_{i}\right) / \mathrm{k}_{\mathrm{B}} T_{i}\right]+1\right]^{-1}$ is the Fermi-Dirac distribution of reservoir $i, f_{M}=\left[\exp \left[\left(\varepsilon-\mu_{M}\right) / \mathrm{k}_{\mathrm{B}} T_{M}\right]+1\right]^{-1}$ is the Fermi-Dirac distribution of reservoir $M, \mathrm{k}_{\mathrm{B}}$ is the Boltzmann constant, $h$ is the Planck constant and $T_{\text {eff } i}(\varepsilon)$ is the effective transmission function of the multi-level quantum dot $Q D_{i}^{4}$

$$
T_{\text {effi, }}(\varepsilon)=\sum_{i=1}^{N} T_{i, N}(\varepsilon)
$$

$T_{i, N}(\varepsilon)=\Gamma_{i}^{2} /\left[\Gamma_{i}^{2}+\left(\varepsilon-\varepsilon_{i, N}\right)^{2}\right]$ is the Lorentz transmission function of Nth energy level in quantum $\operatorname{dot} Q D_{i}, \Gamma_{i}$ is the coupling strength between the quantum dot $Q D_{i}$ and the reservoir next to it. We consider the asymmetry coupling strength, i. e. $\Gamma_{H}=(1+a) \Gamma$ and $\Gamma_{C}=(1-a) \Gamma$, where $a$ is the asymmetry factor which satisfies the condition $-1 \leqslant a \leqslant$ 1 , and $\Gamma$ is the total coupling strength. Utilizing Eqs. (2) and (3), we get the expression of the net heat flux flowing into $M$ reservoir

$$
\begin{aligned}
\dot{Q}_{M M}=\frac{2}{h} \int_{-\infty}^{+\infty} d \varepsilon\left(\varepsilon-\mu_{M}\right) T_{e f f ; H}(\varepsilon)\left[f_{H}(\varepsilon)-f_{M}(\varepsilon)\right] \\
\quad-\frac{2}{h} \int_{-\infty}^{+\infty} d \varepsilon\left(\varepsilon-\mu_{M}\right) T_{e f f, C}(\varepsilon)\left[f_{M}(\varepsilon)-f_{C}(\varepsilon)\right]
\end{aligned}
$$

Because there's a temperature difference between the hot reservoir and the cold reservoir, the heat leakage occurs, i. e. ${ }^{1,2}$

$$
\begin{aligned}
& \dot{Q}_{H M}=\kappa\left(T_{H}{ }^{5}-T_{M}{ }^{5}\right) \\
& \dot{Q}_{M C}=\kappa\left(T_{M}{ }^{5}-T_{C}{ }^{5}\right)
\end{aligned}
$$

where $\kappa$ is defined as the heat leakage coefficient. Then, the COP of the thermal amplifier can be expressed as

$$
\begin{aligned}
\psi & =\frac{\dot{Q}_{M}}{\dot{Q}_{H}+\dot{Q}_{H M}}=\frac{\dot{Q}_{M M}+\dot{Q}_{H M}-\dot{Q}_{M C}}{\dot{Q}_{H}+\dot{Q}_{H M}} \\
& =\frac{\frac{2}{h} \int_{-\infty}^{+\infty} d \varepsilon\left(\varepsilon-\mu_{M}\right) T_{e f f H}(\varepsilon)\left[f_{H}(\varepsilon)-f_{M}(\varepsilon)\right]+\dot{Q}_{H M}-\frac{2}{h} \int_{-\infty}^{+\infty} d \varepsilon\left(\varepsilon-\mu_{M}\right) T_{e f f, C}(\varepsilon)\left[f_{M}(\varepsilon)-f_{C}(\varepsilon)\right]-\dot{Q}_{M C}}{\frac{2}{h} \int_{-\infty}^{+\infty} d \varepsilon\left(\varepsilon-\mu_{H}\right) T_{e f f H}(\varepsilon)\left[f_{H}(\varepsilon)-f_{M}(\varepsilon)\right]+\dot{Q}_{H M}}
\end{aligned}
$$

where $\dot{Q}_{M}$ is also called as the heat-pumping rate (HPR). It is found from Eqs. (5) and (8) that the HPR and the COP of the thermal amplifier are the functions of the central energy levels $\varepsilon_{H}$ and $\varepsilon_{C}$ of two quantum dots, the temperatures $T_{H}$, $T_{M}$ and $T_{C}$, the chemical potentials $\mu_{H}, \mu_{M}$ and $\mu_{C}$ of three electronic reservoirs, the asymmetry factor $a$, the heat leakage coefficient $\kappa$, the total coupling strength $\Gamma$, the number of energy levels $N$ and the energy-level spacing $\delta \varepsilon$.

As a thermal amplifier, the working region can be obtained by deciding the signs of the four thermodynamic parameters $\dot{Q}_{H}, \dot{Q}_{M}, \dot{Q}_{C}$ and $\psi$, and they satisfy

$$
\dot{Q}_{H}>0, \dot{Q}_{M}>0, \dot{Q}_{C} \leqslant 0,1 \leqslant \psi \leqslant \psi r
$$

where $\psi_{r}=\frac{T_{M}\left(T_{C}-T_{H}\right)}{T_{H}\left(T_{C}-T_{M}\right)}$ is a reversible COP of the thermal amplifier. $^{19}$

\section{Results and Discussions}

\subsection{The Performance Characteristics}

By using Eqs. (3) - (9), one can numerically plot the threedimensional (3D) projection graphs of the HPR $\dot{Q}_{M}$ and the COP $\psi$ varying with $\varepsilon_{C}-\mu_{C}$ and $\varepsilon_{H}-\mu_{H}$ at asymmetric factor $a$, as shown in Fig. 2, where $\varepsilon_{C}-\mu_{C}$ and $\varepsilon_{H}-\mu_{H}$ are the relative positions of the central energy levels in quantum dots $Q D_{C}$ and $Q D_{H}$. It is seen from Fig. 2 that both HPR and COP increase as the asymmetric factor increases and there exist the maximum HPR and COP at some optimal values of $\varepsilon_{C}-\mu_{C}$ and $\varepsilon_{H}-\mu_{H}$. And the working regions of the thermal amplifier increase as the asymmetric factor increases.

Similarly we can numerically plot the threedimensional (3D) projection graphs of the HPR $\dot{Q}_{M}$ and the COP $\psi$ varying with $\varepsilon_{C}-\mu_{C}$ and $\varepsilon_{H}-\mu_{H}$ at different number of energy levels $N$, as shown in Fig. 3. It is seen from Fig. 3 that the HPR increases as the number of energy levels increases while the COP decreases as the number of energy levels increases. There exist the maximum HPR and COP at some optimal values of $\varepsilon_{C}-\mu_{C}$ and $\varepsilon_{H}-\mu_{H}$. And the working regions of the thermal amplifier decrease as the number of energy levels increases. When the number of energy levels is large enough, the thermal amplifier loses its 
role. We also plot the three-dimensional (3D) projection graphs of the HPR $\dot{Q}_{M}$ and the COP $\psi$ varying with $\varepsilon_{C}-\mu_{C}$ and $\varepsilon_{H}-\mu_{H}$ at different energy-level spacing $\delta \varepsilon$, as shown in Fig. 4. It is seen from Fig. 4 that the HPR increases as the energy-level spacing increases while the COP decreases as the energy-level spacing increases. There exist the maximum HPR and COP at some optimal values of $\varepsilon_{C}-\mu_{C}$ and $\varepsilon_{H}-\mu_{H}$.
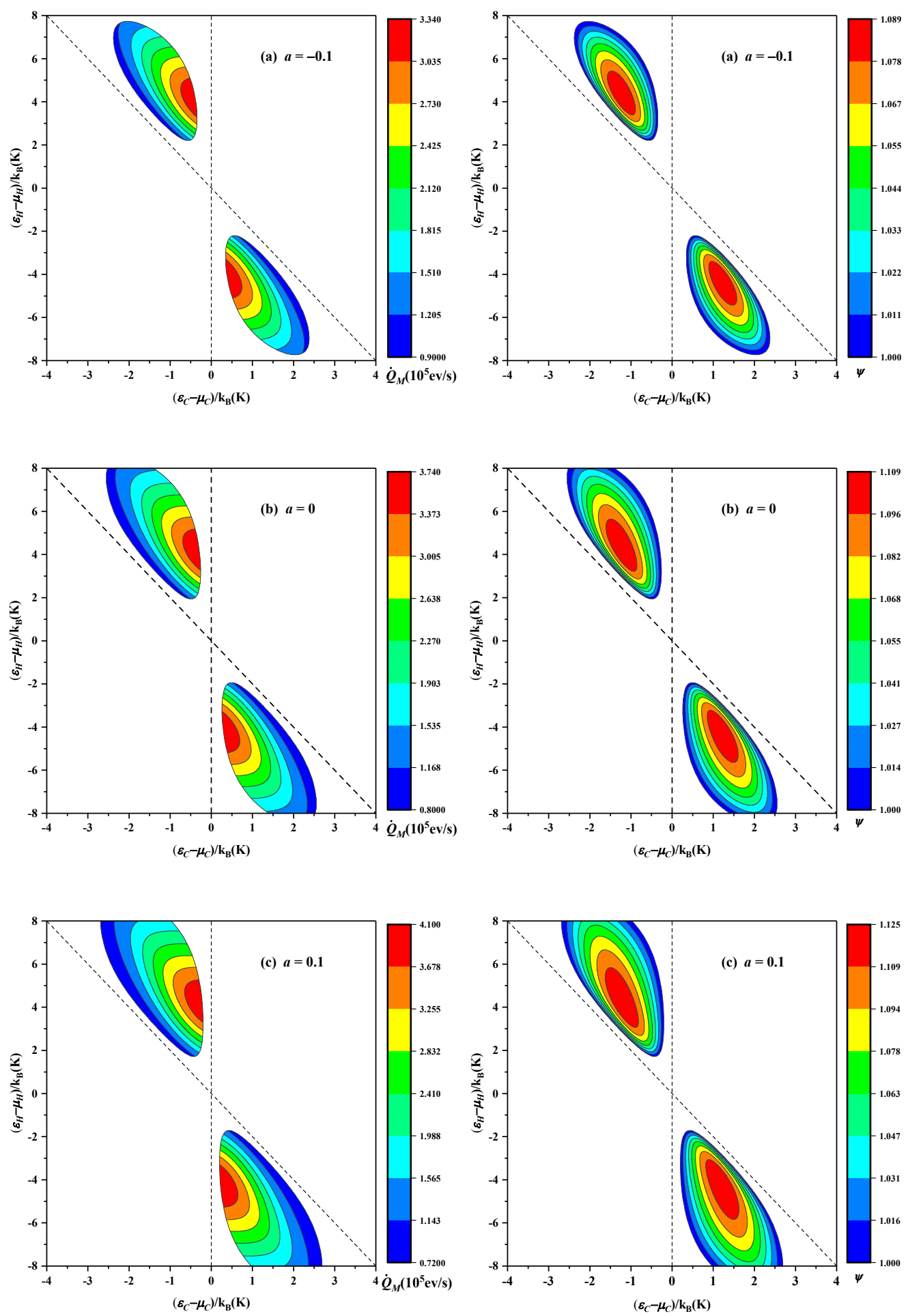

Fig. 2 Left: the three-dimensional projection graphs of the HPR $\dot{Q}_{M}$ versus the relative central energy levels $\varepsilon_{C}-\mu_{C}$ and $\varepsilon_{H}-\mu_{H}$ at different asymmetric factor $a$. Right: the threedimensional projection graphs of the $\mathrm{COP} \psi$ versus the relative central energy levels $\varepsilon_{C}-\mu_{C}$ and $\varepsilon_{H}-\mu_{H}$ at different asymmetric factor $a$. Other parameters are given as $N=1, T_{H}=$ $4 \mathrm{~K}, T_{M}=2 \mathrm{~K}, T_{C}=1 \mathrm{~K}, \Gamma / \mathrm{k}_{\mathrm{B}}=0.1 \mathrm{~K}, \delta \varepsilon / \mathrm{k}_{\mathrm{B}}=0 \mathrm{~K}$ and $\kappa=0$. 

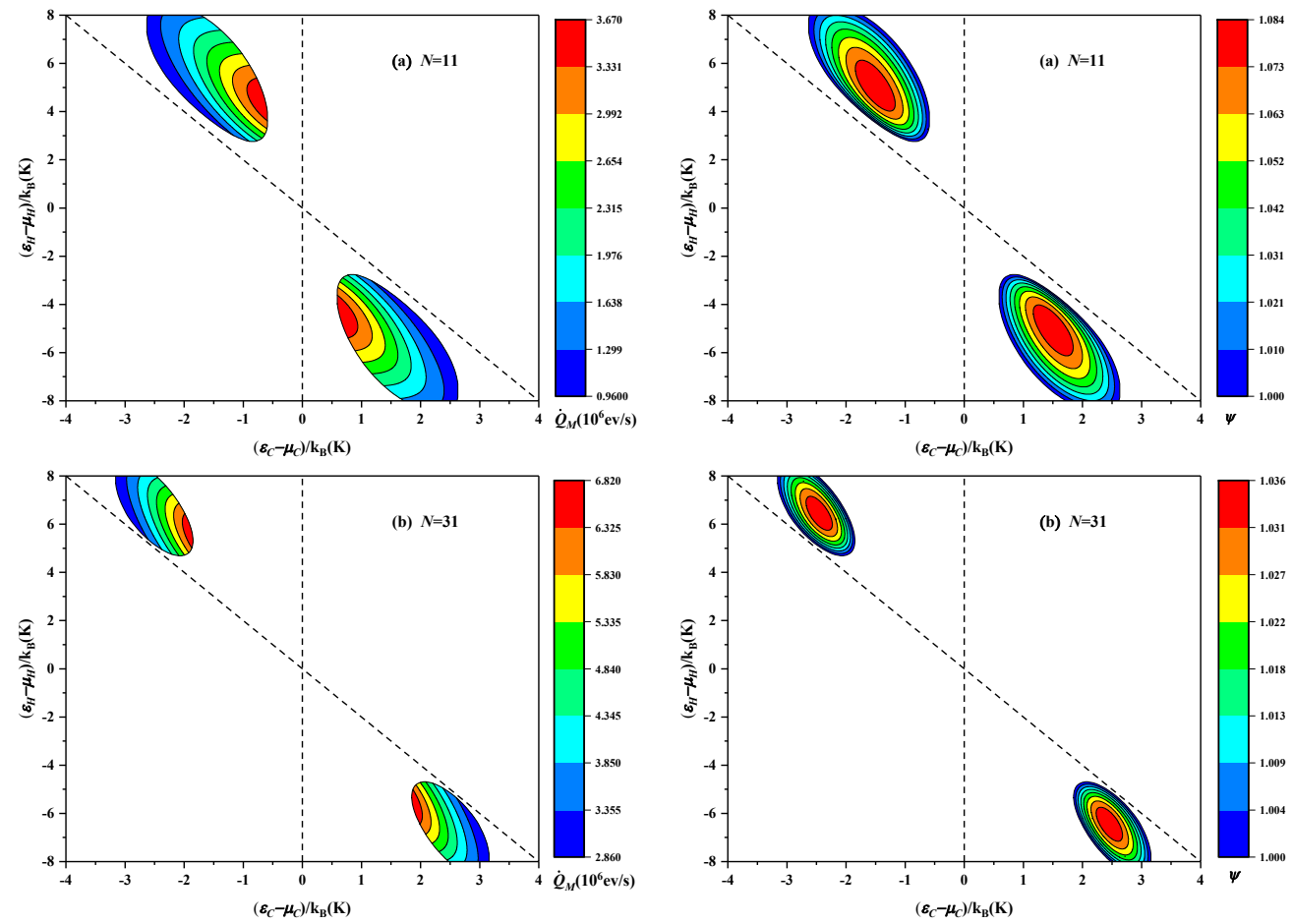

Fig. 3 Left: the three-dimensional projection graphs of the HPR $\dot{Q}_{M}$ versus the relative central energy levels $\varepsilon_{C}-\mu_{C}$ and $\varepsilon_{H}-\mu_{H}$ at different number of energy levels $N$. Right: the three-dimensional projection graphs of the COP $\psi$ versus the relative central energy levels $\varepsilon_{C}-\mu_{C}$ and $\varepsilon_{H}-\mu_{H}$ at different number of energy levels $N$. Here $\delta \varepsilon / \mathrm{k}_{\mathrm{B}}=0.1 \mathrm{~K}$ and $a=0$, the other parameters are the same as those used in Fig. 2.
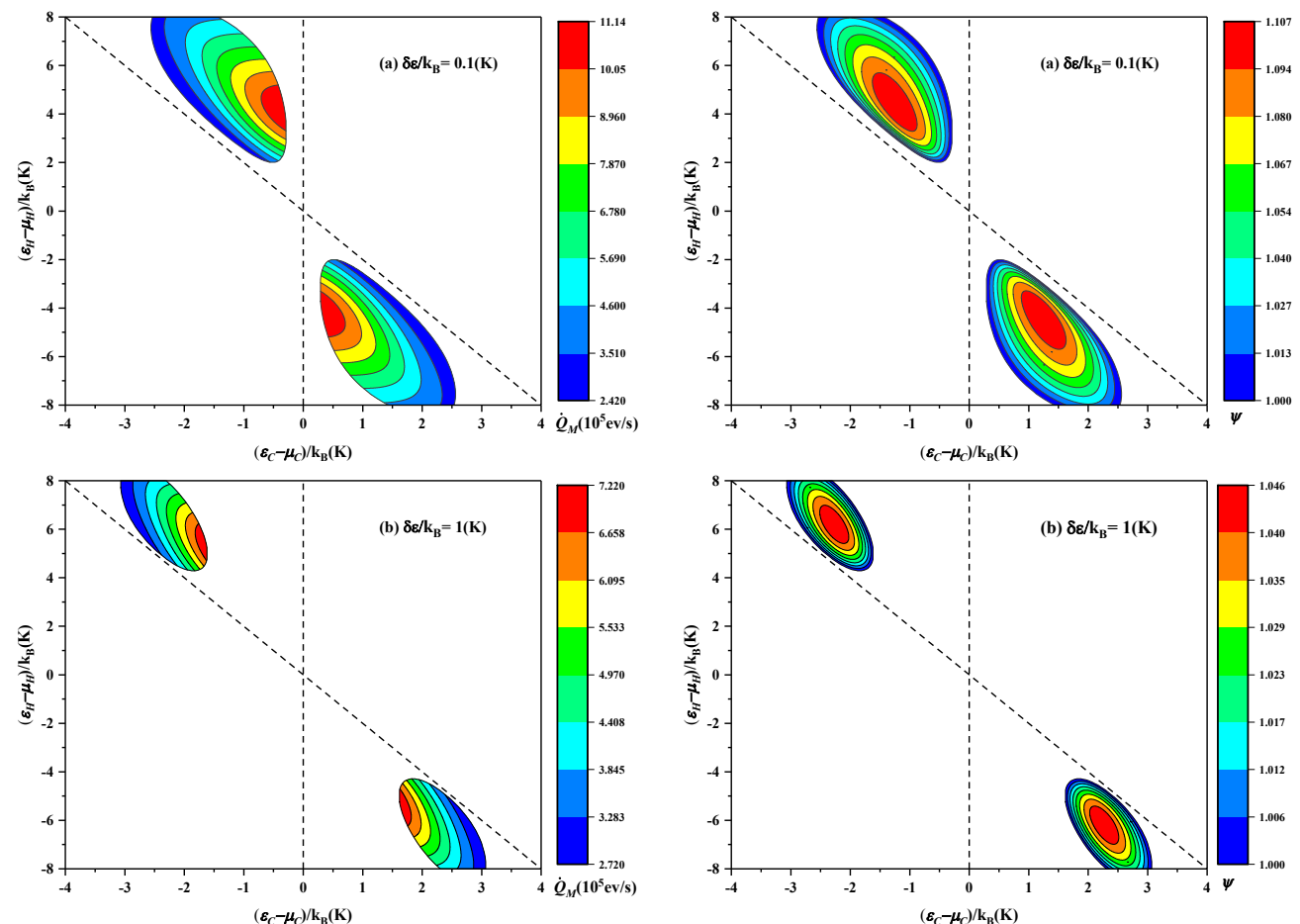

Fig. 4 Left: the three-dimensional projection graphs of the HPR $\dot{Q}_{M}$ versus the relative central energy levels $\varepsilon_{C}-\mu_{C}$ and $\varepsilon_{H}-\mu_{H}$ at different energy-level spacing $\delta \varepsilon$. Right: the three-dimensional projection graphs of the $\mathrm{COP} \psi$ versus the relative central energy levels $\varepsilon_{C}-\mu_{C}$ and $\varepsilon_{H}-\mu_{H}$ at different energy-level spacing $\delta \varepsilon$. Here $N=3$ and $a=0$, the other parameters are the same as those used in Fig. 2. 


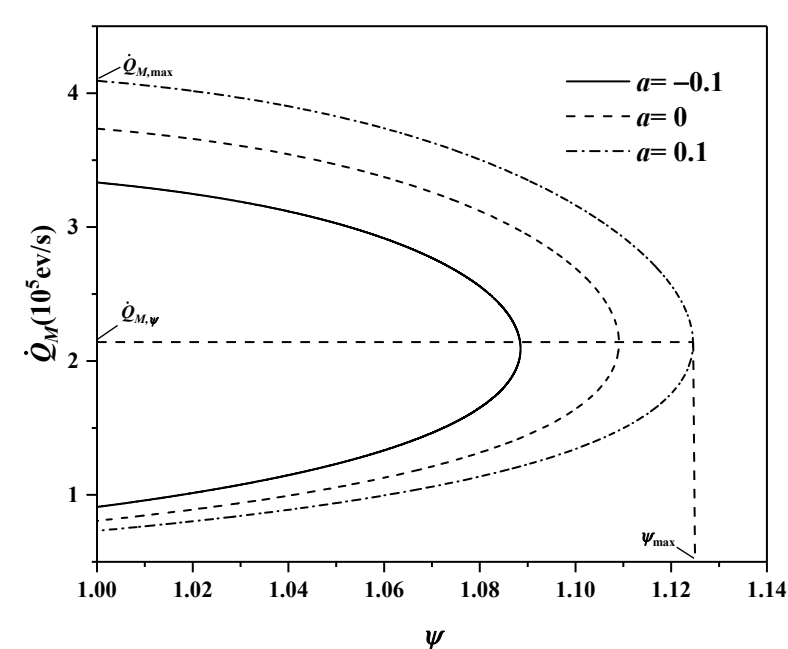

Fig. 5 The performance characteristic curves between the HPR and the COP at different asymmetric factor $a$. The other parameters are the same as those used in Fig. 2.

Further, we plot the characteristic curves between the HPR and the COP at different asymmetric factor $a$, as shown in Fig. 5. It is found from Fig. 5 that there are the maximum COP $\psi_{\text {max }}$ and the maximum HPR $\dot{Q}_{M \text {, max }}$, and they increase as asymmetric factor increases. The corresponding HPR $\dot{Q}_{M . \psi}$ at the maximum COP is almost unchanged. The reasonable optimal region should be in the upper half part of the curve, i.e. $\dot{Q}_{M, \psi} \leqslant \dot{Q}_{M} \leqslant \dot{Q}_{M \text {, max }}$.

Similarly, we plot the characteristic curves between the HPR and the COP at different heat leakage coefficient $\kappa$, as shown in Fig. 6. It is found from Fig. 6 that there are a maximum COP $\psi_{\text {max }}$, whose corresponding HPR $\dot{Q}_{M, \psi}$ and a maximum HPR $\dot{Q}_{M, \max }$. The maximum HPR $\dot{Q}_{M, \max }$ increases as the heat leakage coefficient $\kappa$ increases. While the maximum COP $\psi_{\max }$ and its corresponding PHR $\dot{Q}_{M, \psi}$ decrease as the heat leakage coefficient $\kappa$ increases.

\subsection{The Analysis of Optimal Performance}

Using Eqs. (5) - (8) and the extremal conditions

$$
\begin{gathered}
\frac{\partial \dot{Q}_{M}}{\partial\left(\varepsilon_{C}-\mu_{C}\right)}=0, \frac{\partial \dot{Q}_{M}}{\partial\left(\varepsilon_{H}-\mu_{H}\right)}=0 \\
\frac{\partial \psi}{\partial\left(\varepsilon_{C}-\mu_{C}\right)}=0, \frac{\partial \psi}{\partial\left(\varepsilon_{H}-\mu_{H}\right)}=0
\end{gathered}
$$

we can obtain the maximum HPR $\dot{Q}_{M, \text { max }}$ and maximum COP $\psi_{\max }$, as well as the corresponding HPR $\dot{Q}_{M, \psi}$ at the maximum COP and the corresponding COP $\psi_{\dot{\varphi}_{u}}$ at the maximum HPR. The curves of the maximum HPR $\dot{Q}_{M \text {, max }}$ and maximum COP $\psi_{\text {max }}$ versus the number of energy levels $N$ are plotted by numerical calculation, as shown in Fig. 7 .

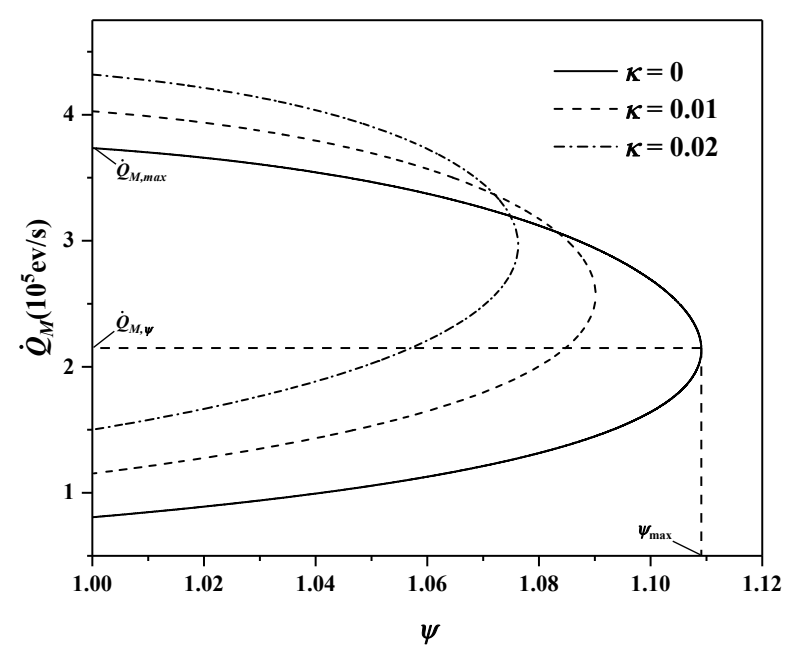

Fig. 6 The performance characteristic curves between the HPR and the COP at different heat leakage coefficient $\kappa$. The other parameters are the same as those used in Fig. 2.

And the curves of the maximum HPR $\dot{Q}_{M \text {, max }}$ versus the energy level spacing $\delta \varepsilon$ are plotted, as shown in Fig. 8. It is seen from Fig. 7 that $\dot{Q}_{M, \max }$ is a monotonically increasing function of $N$. When $N$ is small, the maximum HPR $\dot{Q}_{M \text {, max }}$ almost increases linearly. The value of $\dot{Q}_{M, \max }$ approaches the saturation at large $N$ because the energy levels far from Fermi level are not involved in resonant tunneling. While the maximum COP is a monotonically decreasing function of $N$. When $N=72$, the thermal amplifier almost reaches the minimum value $\psi=1$. In such a case, the heat flows out from the reservoir $H$ is equal to the heat flows into the reservoir $M$. It is seen from Fig. 8 that the maximum HPR decreases as the energy level spacing $\delta \varepsilon$ increases. The denser discrete energy level is, the larger the HPR is.

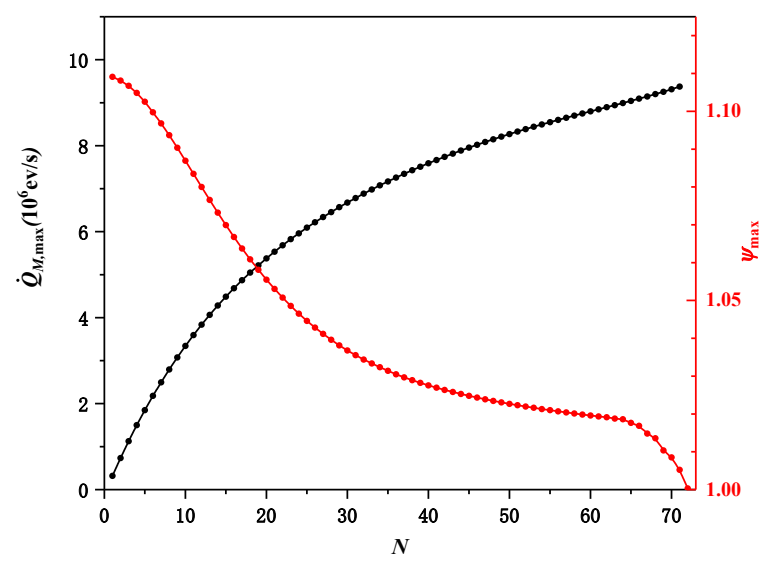

Fig. 7 The maximum HPR $\dot{Q}_{M, \text { max }}$ and maximum COP $\psi_{\text {max }}$ versus the number of energy levels $N$, where $a=0$ and $\delta \varepsilon / \mathrm{k}_{\mathrm{B}}=0.1 \mathrm{~K}$. The other parameters are the same as those used in Fig. 2. 


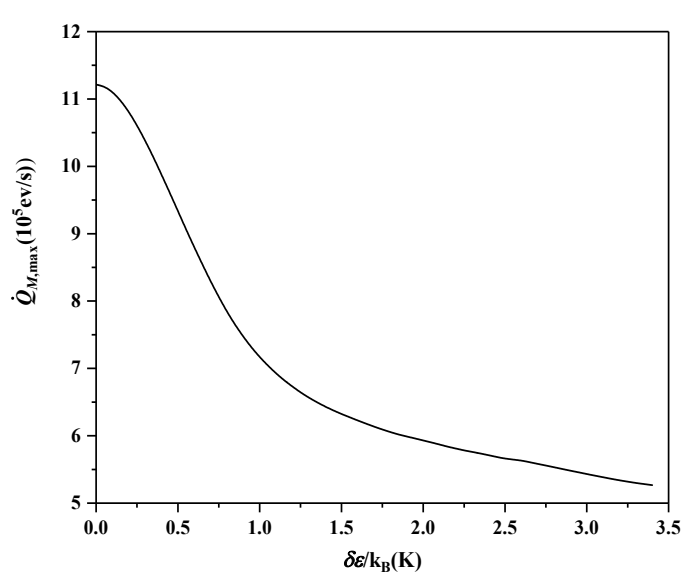

Fig. 8 The maximum HPR $\dot{Q}_{M \text {,max }}$ versus the energy level spacing $\delta \varepsilon$, where $N=3$ and $a=0$. The other parameters are the same as those used in Fig. 2.

Similarly the curves of the maximum HPR $\dot{Q}_{M, \max }$ and the maximum COP $\psi_{\text {max }}$ versus the asymmetric factor $a$ are plotted at different heat leakage coefficient $\kappa$, as shown in Fig. 9 and Fig. 10. It is found from Fig. 9 that the maximum HPR $\dot{Q}_{M, \text { max }}$ firstly increases then decreases as the asymmetric factor increases and reaches its maximum value at $a \approx 0.53$. Moreover, the maximum HPR $\dot{Q}_{M \text { max }}$ increases as the heat leakage coefficient increase. But the corresponding asymmetric factor at the maximum value of $\dot{Q}_{M, \text { max }}$ slightly decreases with the heat leakage coefficient. In Fig. 10, the maximum COP $\psi_{\text {max }}$ firstly increases then decreases as the asymmetric factor increases and reaches its maximum value at $a \approx 0.4$. The maximum value of $\psi_{\max }$ and its corresponding asymmetric factor decrease with the increase of $\kappa$. Thus, in order to obtain the optimal performance of the thermal amplifier, including both larger HPR and COP, the asymmetric factor should be set in the region $0.4 \leqslant a \leqslant 0.53$.

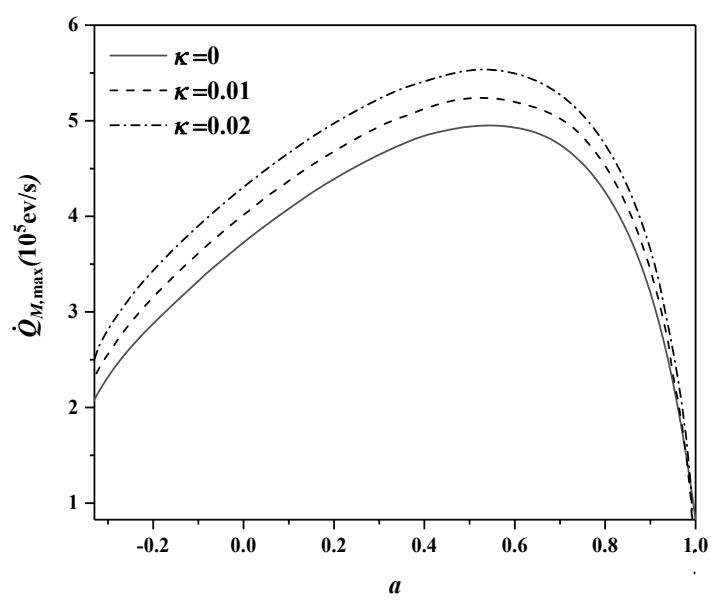

Fig. 9 The curves of the maximum HPR $\dot{Q}_{M \text {,max }}$ versus the asymmetric factor $a$ at different heat leakage coefficient $\kappa$. The other parameters are the same as those used in Fig. 2.

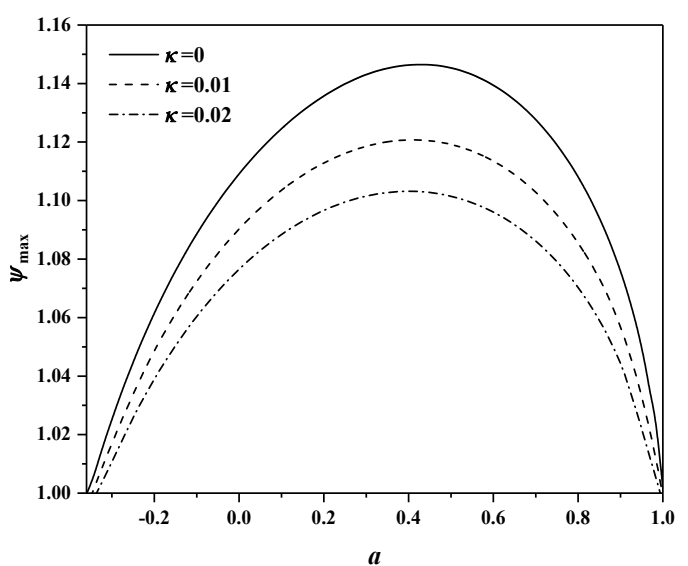

Fig. 10 The curves of the maximum COP $\psi_{\max }$ versus the asymmetric factor $a$ at different heat leakage coefficient $\kappa$. The other parameters are the same as those used in Fig. 2.

\section{Conclusions}

In this paper, we have proposed the three-terminal multilevel quantum dot thermal amplifier with the heat leakage. The influence of the energy-level spacing, the number of energy levels, the total coupling strength, the asymmetry factor and the heat leakage coefficient on the optimal performance parameters has been analyzed in detail. It is concluded that: (a) Choosing an appropriate value of the asymmetric factor can enhance the heat-pumping rate and the coefficient of performance; (b) The addition of the heat leakage can increase the heat-pumping rate, while decrease the coefficient of performance; (c) The denser discrete energy levels in a quantum dot can produce a greater heatpumping rate. These results obtained here may provide some theoretical guidance for the optimal design and operation of practical thermal amplifiers and promote the applications of some heat conversion devices in thermoelectric field.

\section{Conflict of Interest}

There are no conflicts to declare.

\section{Acknowledgements}

We thank the support of the National Natural Science Foundation of China (Grant No. 11875034, 11365015).

\section{References}

1. H. L. Edwards, Q. Niu and A. L. De Lozanne, Appl. Phys. Lett., 1993, 63, 1815.

2. H. L. Edwards, Q. Niu, G. A. Georgakis and A. L. De Lozanne, Phys. Rev. $B, 1995, \mathbf{5 2}, 5714$.

3. J. R. Prance, C. G. Smith, J. P. Griffiths, S. J. Chorley, D. Anderson, G. A. C. Jones, I. Farrer and D. A. Ritchie, Phys. Rev. Lett., 2009, 102, 146602.

4. A. N. Jordan, B. Sothmann, R. Sánchez and M. Büttiker, Phys. Rev. B, 2013, 87, 075312 . 
5. B. Sothmann, R. Sánchez, A. N. Jordan and M. Büttiker, New J. Phys., 2013, 15, 095021.

6. Y. Choi and A. N. Jordan, Phys. E, 2015, 74, 465.

7. J. H. Jiang, O. Entin-Wohlman, Y. Imry, Phys. Rev. B, 2012, 85, 075412.

8. J. H. Jiang and Y. Imry, Phys. Rev. B, 2018, 97, 125422.

9. Z. M. Ding, L. G. Chen, Y. L. Ge and Z. H. Xie, Sci. China: Tech. Sci., 2019, 62, 337.

10. S. S. Qiu, Z. M. Ding, L. G. Chen, F. K. Meng and F. R. Sun, Eur. Phys. J. Plus, 2019, 134, 273.

11. G. Z. Su, Y. C. Zhang, L. Cai, S. H. Su and J. C. Chen, Energy, 2015, 90, 1842.

12. Y. C. Zhang, G. X. Lin and J. C. Chen, Phys Rev E, 2015, 91, 052118.

13. R. Q. Wang, J. C. Lu, C. Wang and J. H. Jiang, Sci. Rep., 2018, 8, 2607.

14. J. C. Chen and Z. Yan, J. Chem. Phys., 1989, 90, 4951.

15. L. G. Chen et al, Energy Convers Manage, 1989, 38, 727.

16. M. H. Ahmadi, M. A. Ahmadi, F. Pourfayaz and M. Bidi, Energy Convers Manage, 2016, 110, 260.

17. S. Z. Wu, G. X. Lin and J. C. Chen, Renewable Energy, 2005, 30, 2257.

18. S. H. Su, Y. C. Zhang, J. C. Chen and T. M. Shih, Scientific Reports, 2016, 6, 21425.
19. W. L. Peng, T. J. Liao, Y. C. Zhang, G. Z. Su, G. X. Lin and J. C. Chen, Energy Convers Manage, 2017, 143, 391.

20. W. L. Peng, Z. L. Ye, X. Zhang and J. C. Chen, Energy Convers Manage, 2018, 166, 74.

21. Z. C. Shi, J. Fu, W. F. Qin and J. Z. He, Chin. Phys. Lett., 2017, 34, 110501.

22. C. Li, Y. Zhang and J. He, Chin. Phys. Lett., 2013, 30, 100501.

23. R. Q. Wang, J. C. Lu, C. Wang and J. H. Jiang, Sci. Rep., 2018, 8, 2607.

24. B. Sothmann and M. Büttiker, Europhys. Lett., 2012, 99, 27001.

25. B. Rutten, M. Esposito and B. Cleuren, Phys. Rev. B, 2009, 80, 235122.

26. B. Cleuren, B. Rutten and C. Van den Broeck, 2012, Phys. Rev. Lett., 108, 120603.

27. Z. C. Shi, J. Z. He and Y. L. Xiao, Sci Sin-Phys. Mech. Astron., 2015, 45, 50502 .

28. C. Li, Y. Zhang, J. Wang and J. He, Phys. Rev. E, 2013, 88, 062120.

29. Y. C. Zhang and J. Z. He, Chin. Phys. Lett., 2013, 30, 010501.

Publisher's Note Engineered Science Publisher remains neutral with regard to jurisdictional claims in published maps and institutional affiliations. 